\title{
Cell-free fetal DNA testing: who is driving implementation?
}

\author{
Jessica Mozersky, $\mathrm{PhD}^{1}$ and Michael T. Mennuti, MD²
}

The introduction of noninvasive cell-free fetal DNA (cffDNA) testing to detect fetal Down syndrome, and several other aneuploidy syndromes, signals a major shift in prenatal screening and diagnostic practice. Its arrival comes 15 years after Lo and colleagues reported the presence of circulating fetal DNA in maternal plasma. It has received much attention, both expectant and cautionary. The cffDNA testing enables prospective parents to obtain information about common survivable fetal aneuploidies with a high level of accuracy and without the risk of a diagnostic procedure. In comparison with other methods of aneuploidy screening, cffDNA testing offers several advantages. It usually involves testing of a single blood sample that may be obtained as early as 10 weeks of gestation and does not require ultrasound data. The cffDNA testing has higher sensitivity and a lower falsepositive rate as compared with other screening methods and is therefore likely to result in less anxiety and fewer invasive tests. Eventual adoption of cffDNA testing as the primary method for aneuploidy screening could greatly streamline the process and result in important benefits to women.

Noninvasive testing for fetal aneuploidy is commercially lucrative, potentially worth up to one billion USD/year. ${ }^{1}$ This has fueled a competitive rush to bring this testing to market. In the past 12 months, three companies have launched cffDNA testing in the United States, and a fourth plans to do so later this year. Sequenom was the first company to market the test, and its MaterniT21 test (Sequenom Center for Molecular Medicine, San Diego, CA) (renamed MaterniT21 PLUS when trisomies 18 and 13 were added) was the only one available between October 2011 and March 2012. In March 2012, Verinata (Verinata Health, Red Wood City, CA) launched the Verifi test, followed by Ariosa's Harmony test (Ariosa Diagnostics, San Jose, CA) in May 2012. All three companies are embroiled in legal battles over patent infringement, prompting concerns that if one company gains a monopoly on testing, it could dictate cost and potentially limit access. ${ }^{1}$ Despite the fact that organizations such as the American Congress of Obstetricians and Gynecologists and the American College of Medical Genetics and Genomics have not issued practice guidelines, some clinicians have begun to selectively offer cffDNA testing as a part of the screening process. Without this guidance, the rapid introduction of cffDNA testing from several competing companies has created practical challenges for clinicians and patients, which are described in this article.
First, how should providers and patients choose from among the available cffDNA tests? All three companies have published validation studies in peer-reviewed journals that coincided with the commercial launch of their tests. ${ }^{2-4}$ Although there are differences in study design and laboratory methods, each reports similarly high sensitivities and low false-positive rates. These studies were conducted on samples from patients undergoing invasive prenatal diagnosis. In two studies, entry was based on specific high-risk criteria, whereas a third involved patients having invasive prenatal diagnosis for "any indication." The sample size in each publication, particularly of aneuploid pregnancies, has been progressively smaller, perhaps due to commercial pressure to rapidly bring the test to market. Performance characteristics of cffDNA testing in clinical practice have not been reported thus far; unfortunately, there is no systematic followup being sought by the laboratories offering the test. Should the provider's confidence in a particular test and selection for clinical use be based solely on critical review of these studies?

Second, what importance should differences in reporting method play in selecting a test? Clinicians need to be mindful that interpretation of the test is based on quantitative analysis of DNA fragments. Although discrimination between affected and euploid fetuses is excellent, each laboratory has established its own "cutoffs" that will necessarily be associated with falsepositive and false-negative results. These different cutoffs make comparison of the performance of each laboratory test difficult. Sequenom reports dichotomous results, i.e., either positive or negative, on the basis of a $z$-score cutoff of 3 . Ariosa uses a priori risk based on maternal age, provides a risk estimate that ranges from $<1 / 10,000$ to a $>99 \%$ chance that the fetus is affected, and arbitrarily defines a positive cutoff as $1 \%$ risk. Verinata's interpretations are reported as aneuploidy detected or not, except for those results with a "normalized chromosome value" $>2.5$ but $<4$. These are reported as "equivocal for the determination of aneuploidy," which results in $100 \%$ sensitivity but at the expense of more results being unclassifiable. A specific risk estimate or clinical guidance for this category is not provided. The frequency of aneuploidies among the small number of patients in each group with unclassified results would justify offering an invasive diagnostic test $(1 / 7,2 / 5$, and $2 / 2$ for trisomies 21 , 18 , and 13 , respectively). These reporting differences could be relevant for patient care because some clinicians or patients may prefer to receive positive/negative results rather than a

${ }^{1}$ Center for the Integration of Genetic Healthcare Technologies, University of Pennsylvania, Philadelphia, Pennsylvania, USA; ${ }^{2}$ Division of Reproductive Genetics, Department of Obstetrics and Gynecology, University of Pennsylvania, Philadelphia, Pennsylvania, USA. Correspondence: Jessica Mozersky (jessica.mozersky@uphs.upenn.edu) 
numerical risk estimate or to avoid an "unclassifiable result" in certain situations.

Third, what role should cost have as a criterion for deciding which test to offer? Patients certainly want to know about outof-pocket costs, which can vary from $\$ 0$ to $\$ 1,900$ depending on the particular test and their insurance coverage. Most patients who are commercially insured are asked to pay between $\$ 200$ and $\$ 500$. In some states, women on Medicaid can access Sequenom's test at no out-of-pocket cost. This leads to an atypical instance in which those on Medicaid have increased, rather than decreased, access to an emerging technology. Given that cost is one of the biggest barriers to access, selecting the test with the lowest cost to patients may be one way of increasing patient access. However, determining what patients will be expected to pay is not straightforward because patient cost is continually shifting as the companies attempt to remain competitive, while reimbursement rates differ by insurer and the plan purchased by an employer. Furthermore, what may be assumed to be a relatively small difference in price, e.g., $\$ 200$ vs. $\$ 235$, could be meaningful to patients. As women and their partners determine whether to undergo testing and which test(s) to have, could basing a decision on cost generate parental guilt or regret?

Fourth, what is an appropriate standard of care during this period of rapid change? For example, if cffDNA is used as the primary screening test, should pretesting ultrasound be recommended to assure a viable singleton pregnancy without an obvious malformation or cystic hygroma? Additional studies about cffDNA testing are constantly emerging and altering the criteria or contexts in which offering the testing may be appropriate. Initially, cffDNA testing was only validated for trisomy 21 . Testing is now also validated for trisomies 18 and 13, with one company offering to report in certain circumstances and another disclosing fetal gender. Limited data on twin pregnancies have been reported and additional data will take time, although one company is already offering testing for twin pregnancies. More data are needed to determine the sensitivity to detect clinically relevant mosaicism. cffDNA testing has only been validated in high-risk populations or those undergoing invasive testing, although studies in low-risk populations are under way. Even among high-risk populations, questions remain about whether testing should be offered only following positive serum and/or ultrasound findings or as the primary screening test.

Finally, who, if anyone, should be the gatekeeper of cffDNA testing? Should it only be available through services that include genetic counseling? Although this would help to ensure adequate informed consent and return of results, it could strain limited genetic counseling resources, especially if testing expands to include other conditions. Alternatively, providers of obstetrical care may order the test without referring the patient for pre- or post-test counseling. In this case, we need to advocate for the availability of adequate information before the decision to have the test so that it is not perceived as one of several routine or recommended prenatal tests rather than being optional and deserving of careful consideration. Similarly, post-test counseling with accurate interpretation and delivery of results, including test limitations in the context of a couple's personal and family history, will be necessary.

The availability of cffDNA testing has the potential to radically alter prenatal screening practice and significantly improve screening for pregnant women. Commercial opportunity has resulted in substantial investment in developing these methods and bringing them to market. One consequence is that extemporaneous factors such as market and consumer forces are driving the utilization and adoption of cffDNA testing. Such factors could, in turn, determine standard of care rather than evidence based on clinical research. ${ }^{5}$ Some providers speculate that other developments, such as prenatal microarrays, may become the more desired options because they currently provide far more information than cffDNA testing. However, cffDNA testing has already been used to detect single-gene disorders and microdeletions, and, most recently, to sequence the entire fetal genome. It may eventually meet standards for diagnostic testing. Determining appropriate standards for laboratory and clinical care and ensuring equitable access are critical. There is also an urgent need for professional guidelines and education and for further research to guide the clinical translation of this rapidly evolving technology. We believe this is the time for professional organizations, research funding agencies, and the academic community to address some of the questions raised in implementing this technology.

\section{DISCLOSURE}

M.T.M. was chair of the three-person independent Scientific Oversight Committee for the clinical validation study of Sequenom's cell-free DNA test by Palomaki et al. ${ }^{2}$ The Scientific Oversight Committee was completely independent from the company. M.T.M. declares no financial or other conflict of interest as a result of this role, which ended with the completion of sample collection. J.M. declares no conflict of interest.

\section{REFERENCES}

1. Hayden EC. Fetal tests spur legal battle. Nature 2012;486(7404):454.

2. Palomaki GE, Kloza EM, Lambert-Messerlian GM, et al. DNA sequencing of maternal plasma to detect Down syndrome: an international clinical validation study. Genet Med 2011;13:913-920.

3. Bianchi DW, Platt LD, Goldberg JD, Abuhamad AZ, Sehnert AJ, Rava RP. Genome-wide fetal aneuploidy detection by maternal plasma DNA sequencing. Obstet Gynecol 2012;119:890-901.

4. Sparks AB, Struble CA, Wang ET, Song K, Oliphant A. Noninvasive prenata detection and selective analysis of cell-free DNA obtained from maternal blood: evaluation for trisomy 21 and trisomy 18. Am J Obstet Gynecol 2012;206 319.e1-319.e9.

5. Wilfond BS, Nolan K. National policy development for the clinical application of genetic diagnostic technologies. Lessons from cystic fibrosis. JAMA $1993 ; 270: 2948-2954$. 